\section{Acute iatrogenic polycythemia induced by massive red blood cell transfusion during subtotal abdominal colectomy}

\author{
David Chiapaikeo, ${ }^{1}$ Pejman Rohani ${ }^{2}$ \\ 1'Department of Surgery, Monmouth \\ Medical Center, Long Branch, NJ; \\ 2Department of Surgery, Jersey Shore \\ University Medical Center, Neptune, NJ, \\ USA
}

\begin{abstract}
A 46 year old man was transfused ten units of packed red blood cells during subtotal colectomy after intraoperative point-of-care testing values demonstrated hemoglobin values less than seven grams per deciliter $(\mathrm{g} / \mathrm{dL})$. A postoperative hemoglobin analyzed in a standard hematologic laboratory revealed a hemoglobin value of $27.8 \mathrm{~g} / \mathrm{dL}$. He underwent emergent red blood cell depletion therapy which decreased his hemoglobin to $7.5 \mathrm{~g} / \mathrm{dL}$. The physiologic consequences of iatrogenic polycythemia caused by massive transfusion during major abdominal surgery must take into account the fluid shifts that interplay between the osmotic load, viscosity of blood, and postoperative third spacing of fluid. Treatment of acute iatrogenic polycythemia can be effectively accomplished by red blood cell depletion therapy. However, fluid shifts caused by massive transfusion followed by rapid red cell depletion produce a unique physiologic state that is without a welldescribed algorithm for management.
\end{abstract}

\section{Introduction}

Polycythemia is considered to be either a genetic disorder of hematopoietic cells or an adaptive response to chronic medical disease. Polycythemia can also result from medical error, caused by over-transfusion. This can occur when point-of-care testing is used. Morbidity and mortality in this disease process is related to thromboembolic events, leading to end organ damage in the form of cerebrovascular accidents, myocardial infarction, and acute renal failure. Iatrogenic acute polycythemia caused by massive transfusion of red blood cells following major abdominal surgery is exceedingly rare, and its physiologic consequences have not been well described in the literature.

\section{Case Report}

A 46 year old male with a history of mental retardation, cerebral palsy, and seizure disorder, developed Clostridium difficile pancolitis following treatment for pneumonia with antibiotics several weeks prior. He was initially managed medically, but developed an increasing leukocytosis, lactic acidosis, fever curve, and abdominal distension. A computed tomography scan of the abdomen demonstrated a new high-grade small bowel obstruction, ascites, and inflammatory changes involving the entire colon. With these radiologic findings combined with signs and symptoms of oliguric renal failure, hemodynamic instability, increasing tachypnea, the differential diagnosis included toxic megacolon and abdominal compartment syndrome. The patient was brought to the operating room for exploratory laparotomy and the small bowel was decompressed through the appendiceal orifice via an appendectomy. The abdomen was left open following this operation. Two days later, returned to the operating room for subtotal colectomy and end ileostomy for definitive management of the pancolitis. During a six hour operation in which the anesthesia team obtained multiple point-of-care testing (POCT) values intraoperatively demonstrating a hemoglobin below seven grams per deciliter ( $\mathrm{g} / \mathrm{dL})$, he was transfused ten units of packed red blood cells, four units of fresh frozen plasma, and given ten liters of crystalloid with an estimated blood loss of two to three liters. Analyzed in a standard laboratory, a postoperative hemoglobin returned $27.8 \mathrm{~g} / \mathrm{dL}$. The patient was hypothermic, hemodynamically labile requiring the support of vasoactive agents, and had diffuse mottling of the skin with anasarca in addition to multiple electrolyte abnormalities.

Hematology was emergently consulted and arrangements for red blood cell depletion therapy were made. Follow apheresis, his hemoglobin value was $7.5 \mathrm{~g} / \mathrm{dL}$. He required additional blood product transfusions for anemia, thrombocytopenia, and coagulopathy during his intensive care unit course. After resolution of his acute polycythemia and relative stabilization of his hematologic parameters, he was taken back to the operating room for re-exploration. Intra-abdominally, multiple full thickness perforations were found around the ileostomy and abscesses were drained. An abdominal washout was performed, the ileostomy revised, necrotic tissue debrided, a biologic mesh was sutured to the fascial edges, and vacuum assisted wound closure of the abdominal wall was done.

After several days of titrating vasopressors, transfusing blood products, and supportive care, the patient began to clinically improve. He had return of bowel function as evidenced
Correspondence: David Chiapaikeo, Department of Surgery, Monmouth Medical Center, 300 Second Avenue, Long Branch, NJ 07740, USA. Tel.: +1.732.470.3211 - Fax: +1.732.923.6768. E-mail: dasc1261@gmail.com

Key words: polycythemia, massive transfusion, colectomy, point-of-care test.

Contributions: DC, PR, management of the patient reported in the case postoperatively; DC, manuscript writing, PR manuscript review and editing.

Conflict of interest: the authors declare no potential conflict of interests.

Received for publication: 17 September 2014. Accepted for publication: 24 February 2015.

This work is licensed under a Creative Commons Attribution NonCommercial 3.0 License (CC BYNC 3.0).

(C) Copyright D. Chiapaikeo and P. Rohani, 2015 Licensee PAGEPress, Italy

Hematology Reports 2015; 7:5638

doi:10.4081/hr.2015.5638

by ileostomy output and was started on nasogastric tube feedings. He was extubated, and neurological status improved towards his baseline. However, two days post-extubation, the patient went into acute respiratory distress requiring re-intubation. A chest x-ray demonstrated complete opacification of the right lung field, which was attributed to aspiration. Given his poor prognosis, the patient's status was changed to do not resuscitate, he was placed on a versed and morphine drip, terminally extubated, and transferred to hospice.

\section{Discussion}

The mainstay treatment for polycythemia is phlebotomy to reduce hematocrit and blood viscosity. Traditionally, this is done by periodic phlebotomy for patients when they begin to experience symptoms of hyperviscosity, initially manifesting as vision changes. ${ }^{1}$ Most cases of primary or secondary polycythemia develop over years, the result of a genetic mutation or the body's response to a chronic disease process.

Consequences of over-transfusion can arise from adverse effects intrinsically associated with blood transfusion, as well as those associated with development of a profound polycythemia. Hemolytic reactions, transfusion related acute lung injury, electrolyte abnormalities, coagulopathy, and infectious complications can occur with blood transfusion. ${ }^{2}$ Complications of polycythemia include throm- 
botic events manifesting as cerebrovascular accidents, myocardial infarction, pulmonary embolism, deep venous thrombosis, and splanchnic vessel thrombosis. ${ }^{3}$ Hyperviscosity syndromes are a broad array of clinical syndromes characterized by an increase in circulating plasma proteins or cells. Treatment for symptomatic hyperviscosity syndrome is plasmapheresis utilizing a cell separator. ${ }^{1}$ This involves withdrawal of blood, centrifugation or membrane filtration to separate blood components, isolation and removal of the pathologic constituent, and reinfusion of the blood and fluid back into the patient. ${ }^{4}$

Aggressive fluid resuscitation is associated with disturbances on a molecular level, with dysregulation of intracellular and extracellular osmolarity, cytosolic acidification, inactivation of critical enzymes, release of pro-inflammatory mediators that may trigger multi-organ system dysfunction and failure. Pulmonary complications can occur including edema and acute respiratory distress syndrome. Cardiac complications such as arrhythmias and alterations in cardiac contractility leading to decreased cardiac output are possible. Gastrointestinal issues include increased gut permeability and susceptibility to bacterial translocation, ileus, and anastomotic dehiscence. Neurologic dysfunction can also occur with disturbances in neurotransmitter metabolism. Hematologic abnormalities with alterations in blood viscosity and dilution of coagulation factors are possible. Endocrine irregu- larities with dysglycemia and catabolism can result as well. ${ }^{5}$

The accuracy and reliability of POCT, its utility and role in the management of critically ill patients has been reviewed. Various devices for POCT exist, and among them are the HemoCue (HemoCue America, Brea, CA, USA) and I-Stat (Abbott Laboratories, Abbott Park, IL, USA). HemoCue underestimates hemoglobin concentration by two to five percent and exhibits eight to ten times higher variability when compared with carbon monoxide oximeter. ${ }^{6}$ Discrepancies between hemoglobin values measured by the POCT device and lab were greater than $1 \mathrm{~g} / \mathrm{dL}$ in many cases and capillary HemoCue may not be sufficiently accurate to make a therapeutic decision, such as whether a blood transfusion should be given. ${ }^{7}$ The disadvantages of POCT are related to insufficient training, lack of standardization in the collection of blood samples, and deficiencies in internal and external quality assessment. ${ }^{8}$

\section{Conclusions}

Acute polycythemia caused by massive transfusion can be effectively treated with red blood cell exchange therapy under the supervision of a hematologist. POCT devices should be used with caution, especially in critically ill patients where the margin between benefit and harm is small.

\section{References}

1. Stone M, Bogen S. Evidence-based focused review of the management of hyperviscosity syndrome. Blood 2012;119:2205-8.

2. Maxwell M, Wilson M. Complications of blood transfusion. Contin Educ Anaesth Crit Care Pain 2006;6:225-9.

3. Kaushansky K, Lichtman M, Beutler E, et al. Williams hematology. 8th ed. New York: McGraw-Hill; 2010.

4. Zarkovic M, Kwaan H. Correction of hyperviscosity by aphresis. Semin Thromb Hemost 2003;29:535-42.

5. Cotton B, Guy J, Morris J, Abumrad N. The cellular, metabolic, and systemic consequences of aggressive fluid resuscitation strategies. Shock 2006;26:115-21.

6. Rippman C, Nett P, Popovic D, et al. Hemocue, an accurate bedside method of hemoglobin measurement? J Clin Monit 1997;13:373-7.

7. Mimoz 0, Frasca D, Medard A, et al. Reliability of the HemoCue hemoglobinometer in critically ill patients: a prospective observational study. Minerva Anesthesiol 2011;77:979-85.

8. Briggs C, Kimber S, Green L. Where are we with point-of-care testing in haematology? Br J Haematol 2012;158:679-90. 